\title{
Changes in the mechanical properties of dermal sheep collagen during in vitro degradation
}

\author{
L. H. H. Olde Damink, ${ }^{1}$ P. J. Dijkstra, ${ }^{1}$ M. J. A. Van Luyn, ${ }^{2}$ P. B. Van Wachem, ${ }^{2}$ P. Nieuwenhuis, ${ }^{2}$ and \\ J. Feijen ${ }^{1, *}$ \\ ${ }^{1}$ Department of Chemical Technology, University of Twente, P.O. Box 217, 7500 AE, Enschede; ${ }^{2}$ Department of \\ Histology and Cell Biology, University of Groningen, Oostersingel 69/2, 9713 EZ, Groningen, The Netherlands
}

\begin{abstract}
The changes in tensile strength, elongation at break, and high strain modulus of dermal sheep collagen (DSC) during in vitro degradation using bacterial collagenase were studied. The changes in mechanical properties were compared with the change in weight of the samples as a function of degradation time. DSC was crosslinked with either glutaraldehyde (GA) or hexamethylene diisocyanate (HMDIC). During degradation, the changes in mechanical properties of the N-DSC, H-DSC or G-DSC samples were more pronounced than the changes in the weight of the samples. Of
\end{abstract}

the mechanical properties studied, the tensile strength was most susceptible to degradation of the DSC samples. After $2.5 \mathrm{~h}, \mathrm{~N}$-DSC sampies had lost only $55 \%$ of their initial weight, but the samples had no tensile strength left. Similar results were obtained for $\mathrm{H}$-DSC, which retained no tensile strength after $24 \mathrm{~h}$ degradation, whereas only $45 \%$ of the initial weight was lost. G-DSC lost $3.5 \%$ of its weight after $24 \mathrm{~h}$ degradation, but only $25 \%$ of the initial tensile strength remained. (c) 1995 John Wiley \& Sons, Inc.

\section{INTRODUCTION}

Collagen-based materials have potential use in various applications in surgery because of their good biocompatibility, low immunologic reactivity, and good mechanical properties. These applications include the use in vascular surgery, ${ }^{1}$ in abdominal wall repair ${ }^{2}$ in tendon replacement, ${ }^{3}$ or as an artificial skin. ${ }^{4}$ Because of the rapid enzymatic turnover of collagen in the body, the materials are usually stabilized by chemical or physical crosslinking methods to maintain stability during the desired time period. Both glutaraldehyde $(\mathrm{GA})^{5,6}$ and hexamethylene diisocyanate (HMDIC) ${ }^{7}$ are known as crosslinking agents for collagen.

The degradation behavior of collagen-based biomaterials is usually studied by in vitro degradation tests in which the materials are exposed to enzymes such as bacterial collagenase ${ }^{8}$ or pronase, ${ }^{9}$ or chemicals such as $\mathrm{CNBr} .{ }^{10}$ The resistance against degradation is almost exclusively related to the weight loss of the materials in time, measured either by gravimetric ${ }_{r}{ }^{11}$ colorimetric, ${ }^{9}$ or radiolabeling methods. ${ }^{12}$ However, especially when collagen-based materials are used in load-bearing applications such as in tendon or ab-

*To whom correspondence should be addressed. dominal wall repair, it is more appropriate to monitor the changes in the mechanical properties of the materials-that is, tensile strength and modulus during degradation. ${ }^{13,14}$

In this article, the in vitro degradation of noncrosslinked and crosslinked dermal sheep collagen (DSC) using bacterial collagenase is reported. DSC was crosslinked with either GA (G-DSC) or HMDIC (H-DSC). The susceptibility of the materials toward degradation by bacterial collagenase was related to the changes in tensile strength, elongation at break, and high strain modulus. The change in the mechanical properties was conıpared with the change in weight of the samples as a function of degradation time.

\section{MATERIALS AND METHODS}

\section{Crosslinking of dermal sheep collagen}

Dermal sheep collagen was obtained from the Zuid-Nederlandse Zeemlederfabriek (Oosterhout, The Netherlands) and had been treated as reported previously. ${ }^{15}$ The fibrous collagen network was washed four times with water, two times with ace- 
tone, and two times with water, and subsequently frozen and lyophilized to give noncrosslinked dermal sheep collagen (N-DSC). The collagen thus obtained showed the native banding in TEM and was resistant to trypsine digestion.

Crosslinking of N-DSC samples with glutaraldehyde (G-DSC) was performed by immersion of $\mathrm{N}$-DSC samples in phosphate buffer $(0.054 \mathrm{M}$ $\mathrm{Na}_{2} \mathrm{HPO}_{4}, 0.013 \mathrm{M} \mathrm{NaH} \mathrm{PO}_{4}, \mathrm{pH}$ 7.4) containing $0.5 \%(\mathrm{wt} / \mathrm{vol})$ purified glutaraldehyde (GA) for $1 \mathrm{~h}$ at room temperature to give G-DSC. ${ }^{16} \mathrm{~N}$-DSC samples were crosslinked with hexamethylene diisocyanate (HMDIC) using a 1.5\% (wt/vol) solution of HMDIC in buffer $\left(0.08 \mathrm{M} \mathrm{Na}_{2} \mathrm{HPO}_{4}\right.$ adjusted with $\left.\mathrm{NaOH}\right)$ at $\mathrm{pH}$ 9.5 containing $1.0 \%$ (wt/vol) Tween 20 (z.S.; MerckSchuchardt, Hohenbrunn, FRG) for $5 \mathrm{~h}$ at room temperature to give H-DSC. ${ }^{17}$ After crosslinking, both G-DSC and H-DSC samples were washed two times with distilled water, two times with $4 \mathrm{M} \mathrm{NaCl}$, and four times with distilled water, and subsequently frozen and lyophilized.

\section{Characterization}

Degree of crosslinking

The degree of cross linking of DSC samples with either GA or HMDIC was related to the increase in shrinkage temperature. ${ }^{18}$ Shrinkage temperatures (Ts) of crosslinked or noncrosslinked DSC samples immersed in water were determined using an apparatus similar to that described in IUP/16. ${ }^{19}$ Test specimens were cut $(5 \times 50 \mathrm{~mm})$, mounted, and hydrated for at least $30 \mathrm{~min}$. A heating rate of $2.5^{\circ} \mathrm{C} / \mathrm{min}$ was applied, and the onset of shrinkage was recorded as the Ts.

Free amine group content

The primary amine group content of crosslinked and noncrosslinked DSC samples, expressed as the number of free amine groups present per 1000 amino acids $(n / 1000)$, was determined using $2,4,6-$ trinitrobenzenesulfonic acid (TNBS).$^{20}$ To a sample of 2-4 mg of DSC $1.0 \mathrm{ml}$ of a $4 \%(\mathrm{wt} / \mathrm{vol}) \mathrm{NaHCO}_{3}$ solution and $1.0 \mathrm{ml}$ of a freshly prepared $0.5 \%(\mathrm{wt} / \mathrm{vol}$ ) TNBS (analytic grade; Serva, Heidelberg, FRG) solution in distilled water was added. After reaction for 2 $\mathrm{h}$ at $40^{\circ} \mathrm{C}, 3.0 \mathrm{ml}$ of $6 \mathrm{M} \mathrm{HCl}$ was added and the temperature was raised to $60^{\circ} \mathrm{C}$. Solubilization of DSC was achieved within $90 \mathrm{~min}$. The resulting solution was diluted with $5.0 \mathrm{ml}$ distilled water and the absorbance measured at $345 \mathrm{~nm}$. A control was prepared applying the same procedure, except that $\mathrm{HCl}$ was added before the addition of TNBS. The free amine group content was calculated using a molar absorption coefficient of $14.600 \mathrm{~L} \cdot \mathrm{mol}^{-1} \cdot \mathrm{cm}^{-1}$ for trinitrophenyllysine. ${ }^{21}$ Extraction of the hydrolyzed DSC solution with diethyl ether did not influence the absorbance reading, and was thus omitted.

\section{Mechanical properties}

Stress-strain curves of noncrosslinked and crosslinked DSC samples were determined by uniaxial measurements using an Instron mechanical tester. Tensile test samples $(30.0 \times 6.0 \times 0.8 \mathrm{~mm})$ were cut using a blade knife and were hydrated for at least 30 min in phosphate-buffered saline (PBS; $0.14 \mathrm{M} \mathrm{NaCl}$, $0.01 \mathrm{M} \mathrm{Na}_{2} \mathrm{HPO}_{4}, 0.002 \mathrm{M} \mathrm{NaH}_{2} \mathrm{PO}_{4}, \mathrm{pH} 7.4 ; \mathrm{NPBI}$, Emmercompascuum, The Netherlands) at room temperature. The tensile strength, the elongation at alignment, the elongation at break, the low strain modulus, and the high strain modulus of the sample were calculated from five independent measurements. Because of variations in the mechanical properties of different parts of the sheepskin, ${ }^{22}$ the change in mechanical properties of a crosslinked sample was only compared with the mechanical properties of a matching noncrosslinked control taken from an adjacent part of the skin. Samples used to study the influence of crosslinking on the mechanical properties were always taken from the IUP $/ 2^{23}$ sampling area, parallel to the backbone, and were either crosslinked with HMDIC or GA, or kept as control.

\section{In vitro degradation}

Two different preparations of bacterial collagenase from Clostridium histolyticum (EC 3.4.24.3; Sigma Chemical Company, St Louis, MO) were used. The crude collagenase (no. C-0130) contained $310 \mathrm{U} / \mathrm{mg}$ solid, whereas the purified collagenase (no. C-0773) contained $1790 \mathrm{U} / \mathrm{mg}$ solid (1 U will release peptides from native collagen, equivalent in ninhydrin color to $1.0 \mu \mathrm{mol}$ of $\mathrm{L}$-leucine in $5 \mathrm{~h}$ at $\mathrm{pH} 7.4$ at $37^{\circ} \mathrm{C}$ in the presence of calcium ions).

\section{General procedure}

In a typical degradation experiment, $10 \mathrm{mg}$ samples of noncrosslinked DSC or DSC crosslinked with either GA or HMDIC were immersed in $0.5 \mathrm{ml}$ of a 0.1 $\mathrm{M}$ Tris- $\mathrm{HCl}$ buffer solution ( $\mathrm{pH} 7.4$ ) containing 0.005 $\mathrm{M} \mathrm{CaCl}_{2}$ and $0.05 \mathrm{mg} / \mathrm{ml}$ sodium azide and incubated at $37^{\circ} \mathrm{C}$. After $1 \mathrm{~h}, 0.5 \mathrm{ml}$ collagenase solution in Tris- 
$\mathrm{HCl}$ buffer $\left(37^{\circ} \mathrm{C}\right)$ was added to give the desired final concentration and absolute amount of collagenase (usually $100 \mathrm{U} / \mathrm{ml}, 10 \mathrm{U} / \mathrm{mg}$ DSC). During all degradation experiments the $\mathrm{pH}$ of the buffer remained constant. The degradation was discontinued at the desired time interval by the addition of $0.1 \mathrm{ml} 0.25 \mathrm{M}$ EDTA (Titriplex III p.a.; E. Merck, Darmstadt, FRG). This inhibited at least $92 \%$ of the initial collagenase activity, as was determined using the method of Wünsch. ${ }^{24}$

In preliminary experiments the collagenase activity (100 U/ml) during degradation was monitored. The collagenase activity was not influenced by the presence of sodium azide, but decreased to $90 \%$ of its initial activity after $24 \mathrm{~h}$ incubation at $37^{\circ} \mathrm{C}$. After $48 \mathrm{~h}$ only $70 \%$, and after 6 days only $55 \%$, of the initial collagenase activity was left. Based on these results the collagenase solution was refreshed after a $24-h$ degradation period.

\section{Change in weight}

Two different methods-a spectrophotometric and a gravimetric method--were used to determine the weight loss of DSC samples on degradation. The changes in weight of partially degraded samples were expressed as a percentage of the initial value. The rate of weight loss of the DSC samples was calculated from the initial slope of the weight loss as a function of degradation time and was defined as a percentage of weight loss per hour.

The weight loss was routinely monitored by assaying the supernatant for hydroxyproline using a modification of the method developed by Cheung et al. ${ }^{9} \mathrm{~A}$ known amount of DSC was degraded as described before. After the addition of EDTA, the remaining degradation mixture was centrifuged at $600 \times g$ for 10 min. A $0.1-\mathrm{ml}$ aliquot was transferred into $0.9 \mathrm{ml} 6 \mathrm{M}$ $\mathrm{HCl}$, after which the test tube was evacuated and sealed. The polypeptide fragments present in the aliquot were hydrolyzed at $110^{\circ} \mathrm{C}$ for $20 \mathrm{~h}$. Thereafter, the solvent was evaporated and the hydrolysate was dried for at least $48 \mathrm{~h}$ at $110^{\circ} \mathrm{C}$.

The remaining pellet was dissolved into citric acid/ phosphate buffer $(0.005 \mathrm{M}$ citric acid, $0.01 \mathrm{M}$ $\mathrm{Na}_{2} \mathrm{HPO}_{4}, \mathrm{pH} 6.0$ ) to give a final concentration of hydroxyproline of approximately $6 \mu \mathrm{g} / \mathrm{ml}$. From this solution a $0.6-\mathrm{ml}$ aliquot was reacted with $0.6 \mathrm{ml}$ of a $0.5 \%$ (wt/vol) Chloramine T (p.a.; E. Merck, Darmstadt, FRG) solution in citric acid/phosphate buffer for $15 \mathrm{~min}$ at room temperature. Thereafter, $1.2 \mathrm{ml}$ Ehrlich reagent was added and reacted at $75^{\circ} \mathrm{C}$ for 15 min. The Ehrlich reagent was prepared by dissolving $7 \mathrm{~g}$ of dimethylamino benzaldehyde in $15 \mathrm{ml}$ of $60 \%$ perchloric acid, and was diluted to $100 \mathrm{ml}$ with 2-propanol. After cooling the solution, the absorbance was directly measured at $555 \mathrm{~nm}$ (Uvikon 930 spectrophotometer; Kontron Instruments, Switzerland). A control was prepared by treating $0.6 \mathrm{ml}$ citric acid/ phosphate buffer that contained no hydroxyproline, as described earlier.

The hydroxyproline content was calculated from a calibration curve obtained from a hydroxyproline (for biochemistry, E. Merck, Darmstadt, FRG) solution, which also contained other amino acids present in $\mathrm{DSC}^{25}$ such as glycine, proline, glutamic acid, and alanine. The amount of DSC solubilized on degradation was calculated using a conversion factor based on the hydroxyproline content of nondegraded DSC samples determined using the same procedure as described before.

The weight loss of noncrosslinked or crosslinked DSC samples during degradation as determined using the hydroxyproline assay was validated by a gravimetric determination of the weight loss. Samples were dried overnight under vacuum over $\mathrm{KOH}$ and were weighed. Thereafter, the samples were degraded as described before. After a predetermined degradation period, EDTA was added and the tubes were centrifuged at $600 \times g$ (Sorvall GLC-1, Newtown, CT) for $10 \mathrm{~min}$ and the remaining solution was discarded. The resulting DSC pellet was washed with distilled water and centrifuged. This washing procedure was conducted three times in total. After the final washing step, the remaining pellet was lyophilized and weighed to determine the weight loss of the DSC samples.

\section{Change in mechanical properties}

Changes in mechanical properties were expressed as a percentage of the initial value of either tensile strength, elongation at break, or high strain modulus, respectively. Samples weighing approximately $0.3 \mathrm{~g}$ were degraded using identical weight-to-volume ratios as described earlier. After the degradation was discontinued by the addition of $10 \mathrm{ml} 0.25 \mathrm{M}$ EDTA, the partially degraded samples were washed four times with an ice cold EDTA solution $(1 \mathrm{mM})$ to remove any remaining buffer salts and to inactivate any adsorbed collagenase. Stress-strain curves of partially degraded samples and control samples were determined after hydrating the samples for at least 30 min in PBS containing $1 \mathrm{mM}$ EDTA at room temperature.

\section{RESULTS}

\section{Initial properties}

The results obtained from the Ts measurements and the determination of the free amine group con- 
tent of noncrosslinked DSC (N-DSC) and DSC cross linked with either GA (G-DSC) or HMDIC (H-DSC) are given in Table I. After crosslinking, an increase in Ts and a decrease in the free amine group content for both G-DSC and H-DSC were observed. The Ts of N-DSC was $56^{\circ} \mathrm{C}$. G-DSC samples had a Ts of $78^{\circ} \mathrm{C}$, whereas for $\mathrm{H}-\mathrm{DSC}$ a Ts of $74^{\circ} \mathrm{C}$ was measured. The free amine group content of N-DSC was $33.7 / 1000$ amino acid residues. The free amine group content of G-DSC and H-DSC were 9.9/1000 amino acid residues and 16.9/1000 amino acid residues, respectively.

The mechanical properties of noncrosslinked and crosslinked DSC are given in Table II. No significant differences between the mechanical properties of G-DSC and H-DSC were observed. N-DSC had the same tensile strength and elongation at alignment as G-DSC and H-DSC, but had a lower elongation at break and a lower low strain modulus. The high strain modulus was the highest for N-DSC.

\section{In vitro degradation}

Figure 1a shows the effect of the collagenase concentration at a constant absolute amount of $10 \mathrm{U} / \mathrm{mg}$ DSC in the incubation medium on the degradation of N-DSC. The rate of weight loss of the samples was determined at the initial stage of degradation and is expressed as a percentage of weight loss per hour.

The effect of the absolute amount of collagenase at a constant concentration of $100 \mathrm{U} / \mathrm{ml}$ on the rate of weight loss of DSC is presented in Figure $1 \mathrm{~b}$. A linear correlation between the rate of weight loss and the collagenase concentration was observed and the rate of weight loss appeared not to be influenced by the absolute amount of collagenase. Based on these results, a collagenase concentration of $100 \mathrm{U} / \mathrm{ml}$ and an absolute amount of $10 \mathrm{U} / \mathrm{mg}$ DSC were selected for further experiments.

In vitro degradation experiments were performed using crude collagenase that also contained enzymes such as clostripain, caseinase, and trypsine. To inves- tigate the influence of these contaminating enzymes on the degradation of collagen, purified collagenase that was freed of all enzymatic activity other than collagenase, as specified by the manufacturer, was used in control experiments. Figure $2 a$ gives the results of these experiments. The linear correlation between the percentage of N-DSC solubilized using either crude or purified collagenase shows that the degradation of DSC samples by the contaminating enzymes is negligible or absent. Similar results were found in the case of G-DSC and H-DSC.

To determine whether the diffusion of soluble DSC fragments from the interior of the sample to the supernatant was limited, the following experiment was performed. Two DSC samples of equal weight and thickness were either cut in 60 circular samples $2 \mathrm{~mm}$ in diameter or in six circular samples $7 \mathrm{~mm}$ in diameter. In this way the outer surface area was varied with a factor of 3 . In the case of the $2-\mathrm{mm}$ samples, the average diffusion pathway from the interior of the sample to the supernatant was shorter than in the case of the 7-mm samples. A linear correlation was found between the percentage of N-DSC solubilized in both experiments (Fig. 2b), showing that the measured degradation rate was not dependant on the diffusion of solubilized DSC parts. Results obtained for G-DSC and H-DSC were similar to those found for N-DSC.

To test which method was more appropriate for determining the weight loss of DSC during degradation both a colorimetric method based on the determination of hydroxyproline present and a gravimetric method were used, and the results were compared. Figure $2 c$ shows that a linear relation between both methods was found. The colorimetric method was preferred because this method is easier to perform.

The influence of degradation on the weight and the mechanical properties of N-DSC, G-DSC, and H-DSC as a function of time are given in Figures 3 and $4 a-c$, respectively. The changes are expressed as the percent decrease of the initial value of, respectively, weight, tensile strength, elongation at break, and high strain modulus. Because partially degraded

TABLE I

Shrinkage Temperature and Free Amine Group Content of N-DSC, G-DSC, and H-DSC

\begin{tabular}{|c|c|c|c|c|c|}
\hline Sample & $\begin{array}{c}\text { Crosslink } \\
\text { Concentration } \\
(\% \text { wt } / w t)\end{array}$ & $\begin{array}{l}\text { Crosslink } \\
\text { Time (h) }\end{array}$ & $\mathrm{pH}$ & $\begin{array}{l}\text { Shrinkage } \\
\text { Temperature } \\
\left({ }^{\circ} \mathrm{C}\right)\end{array}$ & $\begin{array}{l}\text { Amine Group } \\
\text { Content }(n / 1000)\end{array}$ \\
\hline N-DSC ${ }^{*}$ & & & & $56.0 \pm 0.3$ & $33.7 \pm 0.3$ \\
\hline $\mathrm{G}-\mathrm{DSC}^{+}$ & 0.5 & 1.0 & 7.4 & $77.7 \pm 0.3$ & $9.9 \pm 0.2$ \\
\hline $\mathrm{H}-\mathrm{DSC}^{\ddagger}$ & 1.5 & 5.0 & 9.5 & $73.8 \pm 0.3$ & $16.9 \pm 0.4$ \\
\hline
\end{tabular}

\footnotetext{
*Noncrosslinked DSC.

${ }^{+}$Glutaraldehyde-crosslinked DSC.

${ }^{\ddagger}$ Hexamethylene diisocyanate crosslinked DSC.

Shrinkage temperatures and amine group contents were measured in triplicate. The free amine group content of DSC is expressed as the number of amine groups present per 1000 amino acid residues.
} 
TABLE II

Mechanical Properties of N-DSC, G-DSC, and H-DSC

\begin{tabular}{lccccc}
\hline Sample & $\begin{array}{c}\text { Tensile Strength } \\
(\mathrm{MPa})\end{array}$ & $\begin{array}{c}\text { Elongation at } \\
\text { Alignment (\%) }\end{array}$ & $\begin{array}{c}\text { Elongation at } \\
\text { Break (\%) }\end{array}$ & $\begin{array}{c}\text { Low Strain } \\
\text { Modulus (MPa) }\end{array}$ & $\begin{array}{c}\text { High Strain } \\
\text { Modulus (MPa) }\end{array}$ \\
\hline N-DSC & $19 \pm 1$ & $68 \pm 6$ & $141 \pm 7$ & $2.0 \pm 0.3$ & $26 \pm 3$ \\
G-DSC $^{+}$ & $18 \pm 1$ & $61 \pm 3$ & $160 \pm 5$ & $4.6 \pm 0.7$ & $16 \pm 1$ \\
H-DSC $^{\ddagger}$ & $17 \pm 2$ & $63 \pm 7$ & $163 \pm 5$ & $3.5 \pm 0.4$ & $16 \pm 1$ \\
\hline
\end{tabular}

*Noncrosslinked DSC.

${ }^{+}$Glutaraldehyde-crosslinked DSC.

†Hexamethylene diisocyanate crosslinked DSC.

All mechanical properties were measured in fivefold and are given as mean \pm standard deviation.

N-DSC behaved like denatured collagen, no dimensions of the test specimens could be measured, and consequently, mechanical properties could not be determined using stress-strain measurements. Therefore, the progress of degradation of N-DSC samples
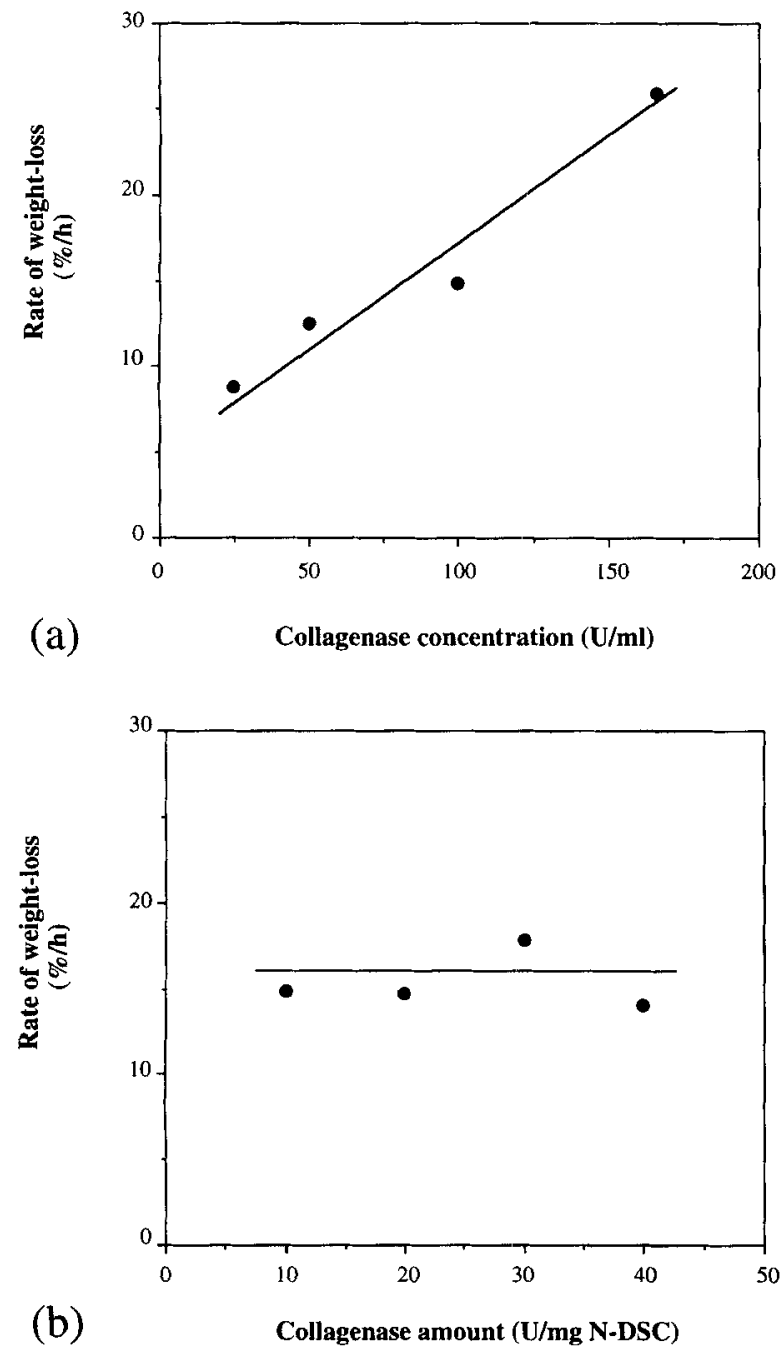

Figure 1. Rate of weight loss of N-DSC as a function of the collagenase concentration ( $a$, absolute amount of collagenase $10 \mathrm{U} / \mathrm{mg} \mathrm{N}$-DSC) and as a function of the absolute amount of collagenase $(b$, collagenase concentration 100 $\mathrm{U} / \mathrm{ml}$ ). The weight loss was measured using the colorimetric $\operatorname{method}(n=3)$. is represented by a dotted line from the start of the degradation to the time at which the samples disintegrated when the degradation tube was vigorously shaken.

Comparing the degradation of N-DSC, G-DSC, and $\mathrm{H}$-DSC, the fastest decrease in weight and change in mechanical properties was observed for N-DSC. N-DSC was found to have a rate of weight loss of $17.6 \% / \mathrm{h}$, as calculated from the initial slope of the curve in Figure 4, and had no mechanical properties left after a 2.5-h degradation period. For H-DSC a rate of weight loss of $1.9 \% / \mathrm{h}$ upon degradation was found, which resulted in a weight loss of $45 \%$ after 24 $\mathrm{h}$ degradation. During this period the change in the mechanical properties of H-DSC was much faster than the change in weight. Only $10 \%$ of the initial tensile strength was left after an 8-h degradation period. The H-DSC samples had no mechanical properties left after a 24 -h degradation period. For G-DSC a rate of weight loss of $0.14 \% / \mathrm{h}$ was found, and only $3.5 \%$ of the initial material was solubilized after $24 \mathrm{~h}$ degradation. During this period a considerable decrease in tensile strength and high strain modulus was observed. Only 25\% of the initial value of the tensile strength and $60 \%$ of the initial value of the high strain modulus were left after a 24-h degradation period. During degradation an almost linear decrease in the elongation at break was found.

\section{DISCUSSION}

The degradation behavior of collagen-based biomaterials is frequently controlled by chemical crosslinking of the materials before application. A controlled degradation rate of the material is important because it may influence the rate of tissue ingrowth, the immunologic response, and the lifetime of the prosthesis after implantation. The resistance of crosslinked, collagen-based materials against degradation is often studied in vitro using enzymes such as bacterial collagenase, and is monitored by changes in weight as a function of exposure time. Changes in the 


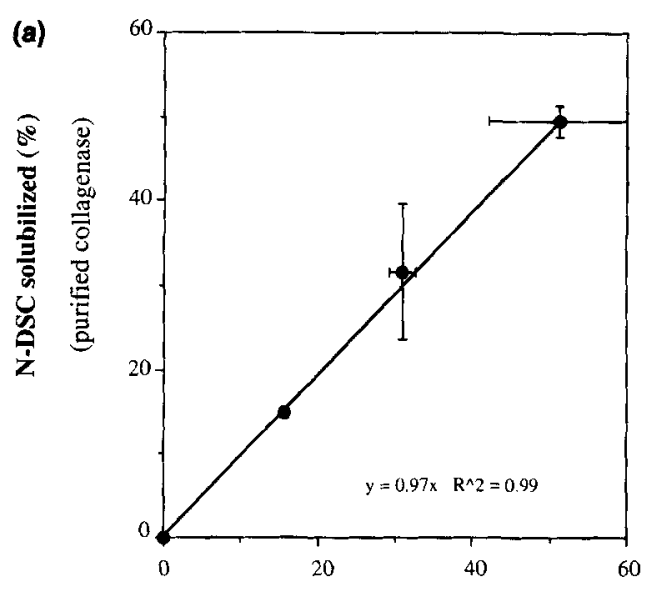

N-DSC solubilized (\%)
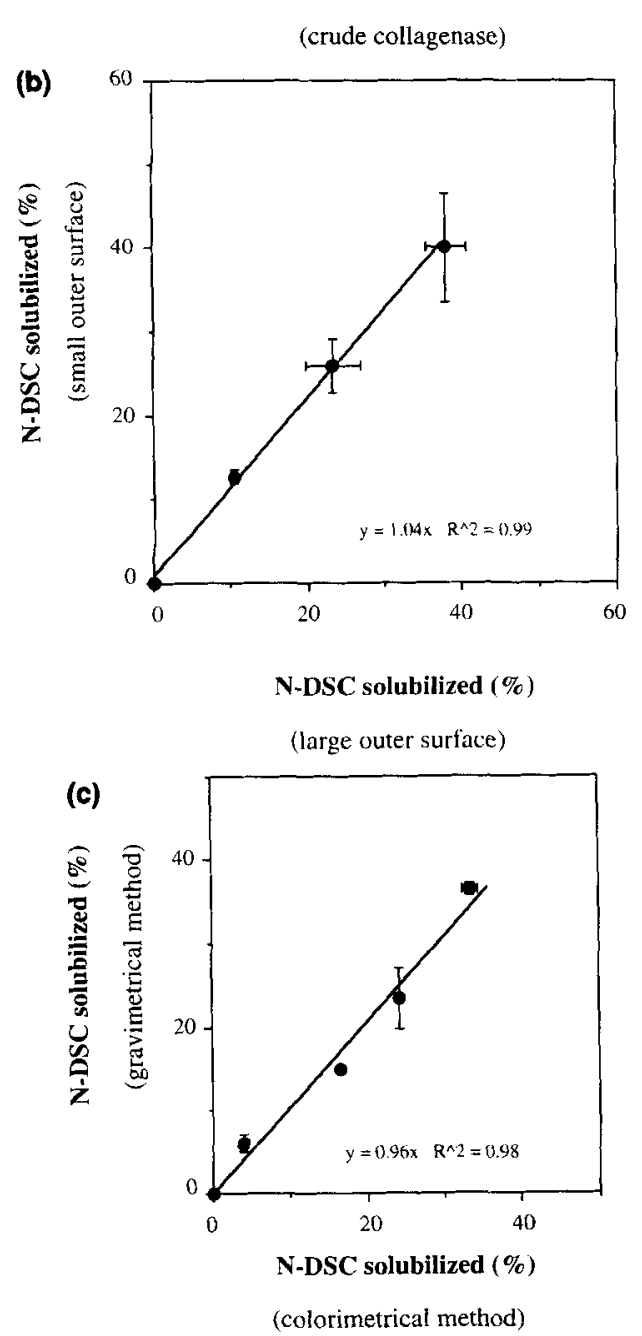

Figure 2. (a) Relation between the percentage of N-DSC solubilized using either purified or crude collagenase. The collagenase concentration and amount were $100 \mathrm{U} / \mathrm{ml}$ and $10 \mathrm{U} / \mathrm{mg}$ N-DSC, respectively, for both crude and purified collagenase. The weight loss was measured using the colorimetric method ( $n=3$ ). (b) Relation between the percentage of N-DSC solubilized using N-DSC samples with either a small or a large outer-surface area. The collagenase concentration and amount were $100 \mathrm{U} / \mathrm{ml}$ and $10 \mathrm{U} / \mathrm{mg} \mathrm{N}$-DSC, respectively. The weight loss was measured using the colorimetric method ( $n=3$ ). (c) Relation between the percentage of N-DSC solubilized determined by either the gravimetric or the colorimetric method. The collagenase concentration and amount were $100 \mathrm{U} / \mathrm{ml}$ and $10 \mathrm{U} / \mathrm{mg} \mathrm{N}$-DSC, respectively $(n=3)$.

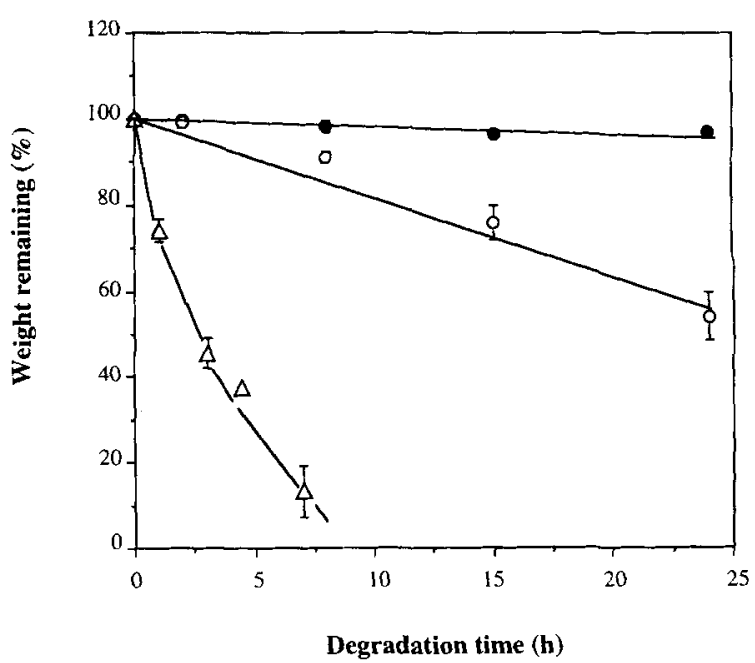

Figure 3. Change in weight as a function of degradation time during exposure of N-DSC (open triangles), H-DSC (open circles), or G-DSC (closed circles) to bacterial collagenase. During degradation a collagenase concentration and amount of $100 \mathrm{U} / \mathrm{ml}$ and $10 \mathrm{U} / \mathrm{mg} \mathrm{DSC}$, respectively, were used $(n=5$, mean $\pm \operatorname{SD})$.

mechanical properties of the materials during implantation become important when the materials are used in load-bearing applications. Therefore, the influence of degradation on the mechanical properties of noncrosslinked and crosslinked DSC was determined. The tensile strength, elongation at break, and high strain modulus of the materials were monitored during exposure to a solution of bacterial collagenase.

Recently, we studied and optimized the crosslinking of DSC with either G-DSC ${ }^{16}$ or H-DSC. ${ }^{17}$ Treatment of N-DSC with these reagents increased the $T$ s and decreased the free amine group content of the material, showing that amine groups are involved during the crosslinking reactions. The highest Ts value and the lowest free amine group content were observed for G-DSC samples. The results presented in Table I are in agreement with these previously reported results. The Ts value of N-DSC was $56^{\circ} \mathrm{C}$. After GA treatment a Ts of $78^{\circ} \mathrm{C}$, and after H-DSC treatment a Ts of $74^{\circ} \mathrm{C}$ was observed, respectively. Furthermore, whereas a free amine group content of 34/ 1000 amino acid residues was found for N-DSC, free amine group contents of 10/1000 and 17/1000 amino acid residues were observed for G-DSC and H-DSC samples, respectively. Although this suggests that G-DSC samples have a higher degree of crosslinking, care has to be taken directly to relate the degree of cross linking to the Ts values of samples crosslinked with different reagents. As discussed previously, the Ts may not only be influenced by the degree of cross linking of the samples, but also by the type of cross link introduced. ${ }^{17}$

The mechanical properties of fibrous collagens such as DSC do not depend solely on the mechanical 

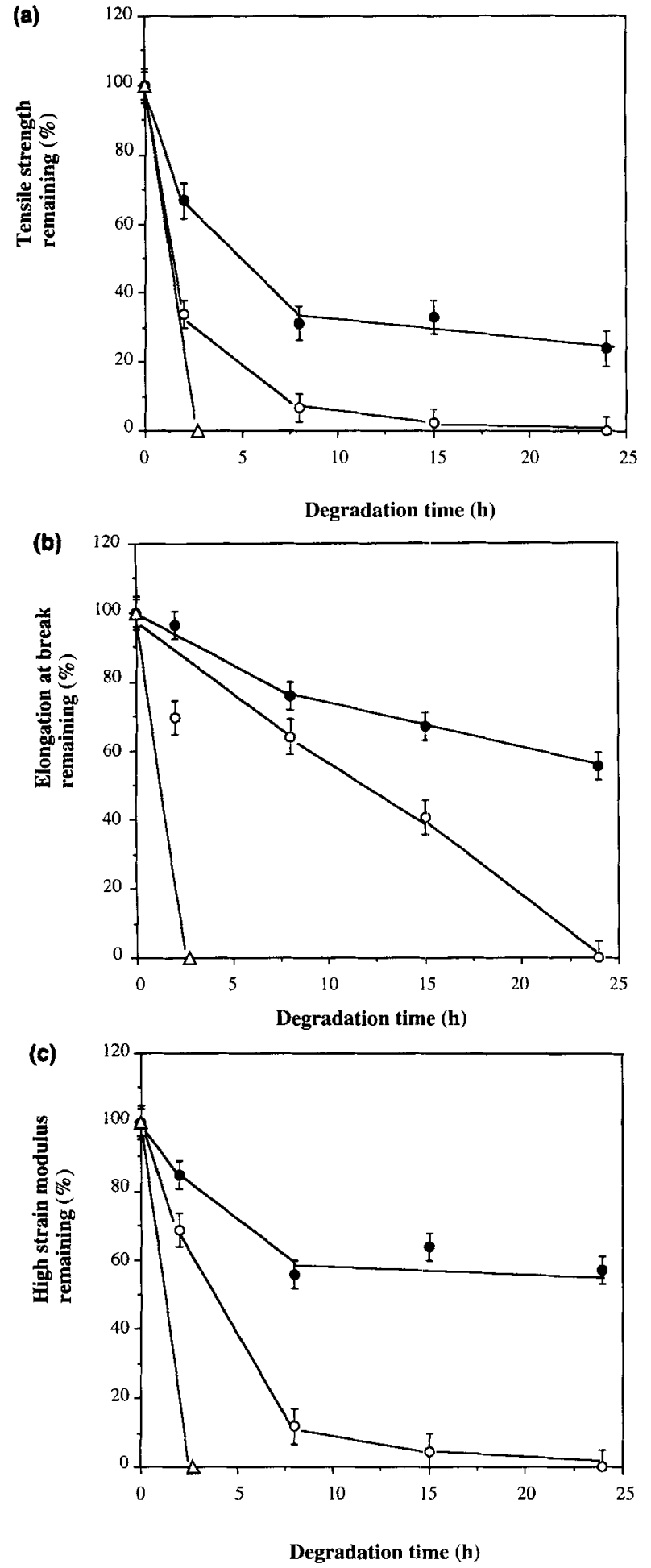

Figure 4. Change in tensile strength (a), change in elongation at break (b), and change in high strain modulus (c) as a function of degradation time during exposure of N-DSC (open triangles), H-DSC (open circles), or G-DSC (closed circles) to bacterial collagenase. During degradation a collagenase concentration and amount of $100 \mathrm{U} / \mathrm{ml}$ and 10 $\mathrm{U} / \mathrm{mg}$ DSC, respectively, were used ( $n=5$, mean $\pm \mathrm{SD})$. properties of the fiber bundles, which are highly structured aggregates of fibers and fibrils. The spatial arrangement of the fiber bundles and the interweaving of fibers from one bundle to another also determine the mechanical properties of the material. The results presented in Table II show that although the tensile strength of both the noncrosslinked and crosslinked DSC samples was similar, the high strain modulus decreased after treatment with GA or H-DSC. These results are consistent with previously reported results, ${ }^{16,17}$ from which it was concluded that crosslinks are introduced only within the fibers. Amine groups located on two adjacent fibers are apparently too far apart to be bridged by the crosslinks. Aligning of the fibers by applying a prestrain to the samples during crosslinking could overcome these steric limitations. ${ }^{16}$

Bacterial collagenase from Clostridium histoliticum was selected as the enzyme for the degradation studies because of its specificity for collagen. These collagenase preparations contain at least six different collagenases, ${ }^{26}$ which have been extensively characterized. ${ }^{27-29}$ The enzymes are capable of cleaving peptide bonds within the triple helical structure and have a specificity for the Pro-X-Gly-Pro-Y region, splitting between $X$ and Gly. ${ }^{24,30}$ Previously, it was shown that cleavage sites within the molecular architecture of aggregates of tropocollagen molecules are not accessible for collagenase, and enzyme-substrate complexes are only formed on the surface of the aggregates. ${ }^{31}$ Similar results have been reported for the enzymatic degradation of microbial polyesters. ${ }^{32}$ The zero-order weight loss of DSC in the initial stage of the degradation reveals that a surface erosion process is involved. However, we cannot distinguish whether the adsorption of collagenase takes place at the fiber bundle surface, fiber surface, or fibril surface.

The initial step during the degradation of DSC involves the formation of an enzyme-substrate complex by adsorption of collagenase onto the collagen molecules. The formation of the enzyme-substrate complex is dependent on the concentration of both the enzyme and the substrate. Thereafter, cleavage of the polypeptide chain is possible, which ultimately will result in the solubilization of DSC fragments. Therefore, the number of enzyme-substrate complexes will determine the rate of weight loss of noncrosslinked collagen samples. Previous studies toward the degradation of noncrosslinked reconstituted collagen using bacterial collagenase ${ }^{33}$ revealed that the rate of weight loss (expressed as a percentage of weight loss per hour) is linearly dependent on the collagenase concentration in the range of $10-200 \mathrm{U} / \mathrm{ml}$. However, using a collagenase concentration of $500 \mathrm{U} / \mathrm{ml}$, a deviation of the degradation rate from this linear relationship occurred. Comparable results have been obtained by others. ${ }^{31}$ 
The results presented in Figure 1a confirm the linear relation between the collagenase concentration and rate of weight loss within the concentration range studied. Because cleavage of the polypeptide chains only occurs after the formation of the enzymesubstrate complex, this indicates that the amount of enzyme-substrate complex is linearly dependent on the collagenase concentration in the concentration range studied. Because an increase in collagenase concentration results in an increase in degradation rate, it is evident that the formation of the enzymesubstrate complex is the rate-limiting step during degradation. Furthermore, this linear relation shows that not all available adsorption sites are involved in the formation of enzyme-substrate complexes. Figure $1 b$ shows that the degradation rate is not influenced by varying the absolute amount of collagenase present per gram of DSC between 10 and $40 \mathrm{U} / \mathrm{mg}$ at a concentration of $100 \mathrm{U} / \mathrm{ml}$. This shows that under these conditions the formation of the enzymesubstrate complex is not limited by the absolute amount of collagenase present in the solution.

To standardize the degradation studies the influence of contaminating enzymes present in the collagenase preparations on the degradation rate were studied. The activity of contaminating enzymes present in the crude collagenase did not influence the weight loss of DSC during degradation, as was shown by the results presented in Figure 2a. This can be because these nonspecific enzymes are not able to degrade the helical part of collagen. ${ }^{34}$ Furthermore, the fraction collagen degraded by these enzymes may be negligible compared with the fraction degraded by collagenase.

In addition, the weight loss during degradation of noncrosslinked or crosslinked DSC may be dependent on the diffusion of DSC fragments solubilized within the matrix of the sample to the supernatant and thus depend on the size of the DSC samples. However, the results presented in Figure $2 b$ show that the size of the DSC samples (i.e., the outer surface area of the sample) does not influence the rate of weight loss measured during degradation, indicating that the transport of degradation products out of the matrix into the supernatant is not limited by diffusion.

From the results obtained during the degradation of N-DSC, H-DSC, and G-DSC samples, it is shown that the mechanical properties (Figs. $4 \mathrm{a}-\mathrm{c}$ ) of the materials are more susceptible to degradation than the weight loss of the materials (Fig. 3). Especially in the initial stage, the influence of degradation on the mechanical properties of the cross linked samples is prominent. After $8 \mathrm{~h}$ degradation only $35 \%$ of the tensile strength and $60 \%$ of the high strain modulus of G-DSC samples were retained. Longer degradation times resulted only in a small additional decrease in both the tensile strength and the high strain modulus
(Fig. 4a and c). Although this might lead to the assumption that both parameters will not decrease any more at longer degradation times, it is expected that these samples will degrade further, eventually resulting in samples with no tensile strength and high strain modulus left.

The mechanical properties of the DSC samples are more susceptible to degradation than the weight loss. Every chain scission brought about by enzymatic action will influence the integrity of the DSC matrix, and thus the mechanical properties of the material. In contrast, a single chain scission does not have to result in the solubilization of a degraded polypeptide fragment. Only after multiple chain scissions have taken place will a change in weight be observed. Furthermore, the interweaving of fibers from one fiber bundle to another is very important for the structural integrity of the fibrous DSC network. These interweaving fibers are predominantly located at the surface of the fiber bundles and are therefore prone to enzymatic attack, especially during the initial stage of the degradation. A decrease in the strength of these fibers will contribute to a larger extent to the changes in the mechanical properties of the DSC samples than changes in the strength of fibers that are not interweaving.

Crosslinking of N-DSC with GA or HMDIC increases the resistance of the material to degradation by collagenase. As is shown in Figures 3 and $4 a-c$, the changes in weight and mechanical properties of the G-DSC and H-DSC samples during collagenase treatment were smaller compared with the changes observed for the N-DSC samples. G-DSC samples were the least affected by degradation. As a result of the introduction of crosslinks in the DSC matrix the cleavage sites on the collagen surface may be less accessible for collagenase. Furthermore, the crosslinks introduced in the material may serve to retain the structure of the samples necessitating on average more chain cleavages before a degraded fragment can be solubilized. As a consequence, this decreases the rate at which other cleavage sites become available for collagenase. Crosslinks may also inhibit the penetration of collagenase in the DSC structure. When, for instance, interfibrillar crosslinks are introduced, the degradation will no longer take place at the fibril surface, but only at the fiber surface. This will substantially reduce the surface area available for adsorption of collagenase.

\section{CONCLUSIONS}

Upon degradation, the change in the tensile strength, elongation at break, and high strain modulus of the N-DSC, H-DSC, and G-DSC samples were more sensitive to degradation than the change in weight. Of the mechanical properties studied, the tensile strength decreased the fastest during cross- 
linking. Both glutaraldehyde and hexamethylene diisocyanate crosslinking increased the resistance of DSC to degradation by bacterial collagenase, as measured by changes in weight and mechanical properties. Samples crosslinked using the former method were most resistant toward degradation by a solution of bacterial collagenase.

\section{References}

1. N. J. Out, P. J. Klopper, K. Breuker, and J. Feijen "Processed dermal sheep collagen as an arterial biograft," Eur. Surg. Res., 16(Suppl 1), 10 (1984).

2. J. S. van der Laan, P. B. van Wachem, J. M. Schakenraad, and P. Nieuwenhuis, "Dermal sheep collagen for the repair of abdominal wall defects," J. Artif. Organs, 14, 661-666 (1991).

3. Y. P. Kato, D. L. Christiansen, R. A. Hahn, S. J. Shieh, J. D. Goldstein, and F. H. Silver, "Mechanical properties of collagen fibers: A comparison of reconstituted and rat tail tendon fibers," Biomaterials, 10, 38-42 (1989).

4. I. V. Yannas, "Biologically active analogues of the extracellular matrix: Artificial skin and nerves," Angew. Chem. Int. Ed. Engl., 29, 20-35 (1990).

5. M. E. Nimni, D. Cheung, B. Strates, M. Kodama, and K. Skeikh, "Chemically modified collagen: A natural biomaterial for tissue replacement," J. Biomed. Mater. Res., 21, 741-771 (1987).

6. E. A. Woodroof, "Use of glutaraldehyde and formaldehyde to process tissue heart valves," $J$. Bioengineering, 2, 1-9 (1978).

7. M. Chvapil, D. Speer, W. Mora, and C. Eskelson, "Effect of tanning agent on tissue reaction to tissue implanted collagen sponge," J. Surg. Res., 35, 402-409 (1983).

8. M. J. White, I. Kohno, A. L. Rubin, and K. H. Stenzel, "Collagen films: Effect of crosslinking on physical and biological properties," Biomat. Med. Dev. Art. Org., 1, 703-715 (1973).

9. D. T. Chueng, N. Perelman, E. C. Ko, and M. E. Nimni, "Mechanism of crosslinking of proteins by glutaraldehyde III. Reaction with collagen in tissues," Conn. Tissue Res., 13, 109-115 (1985).

10. D. T. Cheung and M. E. Nimni, "Mechanism of crosslinking of proteins by glutaraldehyde II. Reaction with monomeric and polymeric collagen," Conn. Tissue Res., 10, 201-216 (1982).

11. D. T. Cheung, N. Perelman, D. Tong, and M. E. Nimni, "The effect of $\gamma$-irradiation on collagen molecules, isolated $\alpha$-chains, and crosslinked native fibers," I. Biomed. Mater. Res., 24, 581-589 (1990).

12. K. A. Mookhtiar, S. K. Mallya, and H. E. Van Wart, "Properties of radiolabeled type I, II and II collagens related to their use as substrates in collagenase assays," Anal. Biochem., 158, 322-333 (1986).

13. C. Huang and I. V. Yannas, "Mechanochemical studies of enzymatic degradation of insoluble collagen fibers," I. Biomed. Mater. Res., 8, 137-154 (1977).

14. C. A. Vater, E. D. Harris, Jr., and R. C. Siegel, "Native cross-links in collagen fibrils induce resistance to human synovial collagenase," Biochem. J., 181, 639645 (1979).

15. T. M. van Gulik, R. A. Christiano, A. H. Broekhuizen, E. L. F. B. Raaymakers, and P. J. Klopper, "A tanned sheep dermal collagen graft as a dressing for split-skin graft donor sites," Neth. J. Surg., 41, 65-67 (1989).

16. L. H. H. Olde Damink, P. J. Dijkstra, M. J. A. van
Luyn, P. B. van Wachem, P. Nieuwenhuis, and J. Feijen, "Glutaraldehyde as a crosslinking agent for collagen-based biomaterials," Mater. Med., submitted.

17. L. H. H. Olde Damink, P. J. Dijkstra, M. J. A. van Luyn, P. B. van Wachem, P. Nieuwenhuis, and J. Feijen, "Crosslinking of dermal sheep collagen using hexamethylene diisocyanate," Mater. Med., submitted.

18. P. J. Flory and R. R. Garrett, "Phase transitions in collagen and gelatin systems," J. Amer. Chem. Soc., 80, 4836-4845 (1958).

19. "IULCS physical test method: Method IUP/16, the measurement of shrinkage temperature," I. Soc. Leather Trades Chemists, 47, 122 (1963).

20. D. L. Gilbert and S. W. Kim, "Macromolecular release from collagen monolithic devices," I. Biomed. Mater. Res., 24, 1221-1239 (1990).

21. C. L. Wang, T. Miyata, B. Weksler, A. L. Rubin, and K. H. Stenzel, "Collagen-induced platelet aggregation and release. I. Effects of side-chain modifications and role of arginyl residues," Biochim. Biophys. Acta, 544, 555-567 (1978).

22. A. Vos and P. J. van Vlimmeren, "Topographic differences in physical properties," J. Soc. Leather Technol. Chem., 57, 93-98 (1973).

23. "Sampling IUP/2," J. Soc. Leather Trades Chemists, 42, 382 (1958).

24. E. Wünsch and H. G. Heindrich, "Zur quantitativen bestimmung der Kollagenase," J. Physiol. Chem., 333, 149-151 (1963).

25. P. Nedkov, R. Glanville, I. Goshev, and K. Kühn, "Isolierung und teilweise Charakterisierung von Schafshautkollagen, in Lösung gebracht mit Hilfe von Subtilisin DY," Leder 34, 54-58 (1983).

26. M. D. Bond and H. E. Van Wart, "Purification and separation of individual collagenases of Clostridium histolyticum using red dye ligand chromatography," Biochemistry, 23, 3077-3085 (1984).

27. M. D. Bond and H. E. Van Wart, "Characterization of the individual collagenases from Clostridium histolyticum," Biochemistry, 23, 3085-3091 (1984).

28. M. D. Bond and H. E. Van Wart, "Relationship between the individual collagenases of Clostridium histolyticum: Evidence for evolution by gene duplication," Biochemistry, 23, 3092-3099 (1984).

29. E. L. Angleton and H. E. Van Wart, "Preparation by direct metal exchange and kinetic study of active site metal substituted class I and class II Clostridium histolyticum collagenases," Biochemistry, 27, 7413-7418 (1988).

30. E. Harper, A. Berger, and E. Katchalski, "The hydrolysis of poly(L-prolyl-glycyl-L-prolyl) by bacterial collagenase," Biopolymers, 11, 1607-1612 (1972).

31. F. S. Steven, "Polymeric collagen fibrils: An example of substrate-mediated steric obstruction of enzymic digestion," Biochim. Biophys. Acta, 452, 151-160 (1976).

32. Y. Doi, Y. Kanesawa, M. Kunioka, and T. Saito, "Biodegradation of microbial copolyesters: Poly(3-hydroxy butyrate-co-3-hydroxyvalerate) and poly(3-hydroxybutyrate-co-4-hydroxybutyrate)," Macromolecules, 23, 26-31 (1990).

33. D. L. Gilbert and S. W. Kim, "Macromolecular release from collagen monolithic devices," I. Biomed. Mater. Res., 24, 1221-1239 (1990).

34. J. Feijen, "Biodegradable polymers for medical purposes," in Polymeric Biomaterials, E. Piskin, and A. S. Hoffman (eds.), Martinus Nijhoff Publishers, Dordrecht, The Netherlands, 1986.

Received December 28, 1993

Accepted July 11, 1994 